\title{
Concept of scanning imaging device for optical telescopes
}

\author{
A.V. Papoyan* \\ Institute for Physical Research, NAS of Armenia, Ashtarak-2, 0203, Armenia
}

\begin{abstract}
We propose an approach of a focal plane imaging device for a telescope, which is based on spatially-scanned solitary photodetector, and can be used as an alternative to matrix detectors (CMOS or CCD). This approach allows to set efficient sensor size, pixel resolution, sensitivity and recording time on customer demand. Technical feasibility of up to $200 \mathrm{pixel} / \mathrm{mm}$ (5000 ppi) spatial resolution for focal field of up to astronomical photographic plate size, with the detection noiseequivalent power of $3 \times 10^{-15} \mathrm{~W} / \sqrt{\mathrm{Hz}}$ and the measurement time of $\approx 1$ minute per megapixel is justified.
\end{abstract}

Keywords: optical astrophotography; telescope focal plane imaging; image sensors

\section{Introduction}

Most of modern detection systems used in optical telescopes are based on two-dimensional matrix (array) light sensors: charge-coupled devices (CCD) or complementary metal-oxide-semiconductors (CMOS), which have successfully replaced photographic plates widely used in past. Matrix sensors with different spatial resolution, sensor size, sensitivity and response time have been developed. Electronic control and digital readout of data for matrix cameras allow implementation of various image processing and enhancement techniques, such as interpolation, filtering, motion detection, etc.

The "parallel" image recording approach used in visual observations, and later in classical and digital photography, is basic, but not unique. An image can also be constructed by sequential pointby-point registration of a light signal. In our previous works, this approach was used to visualize objects in a strongly scattering and absorbing medium (Vardanyan et al. (2010), Vardanyan et al. (2011)). A method was developed and tested in which a laser diode and a photodiode mounted on the same holder spatially moved stepwise along a certain path, covering a rectangular area, and a stationary sample under study was installed between them. The operation of the device was completely computer controlled. The system was designed to be used for biomedical imaging, as well as technological and security screening applications.

The present work analyzes the possibility of using the method of pointwise image formation in astronomical photography.

\section{Scanning technique and algorithm}

The key distinction of the proposed approach from the conventional image recording techniques is the use of a single-pixel spatially-scanned photodetector instead of matrix (array) detector. With this approach, the image is being built as the solitary detector passes step by step across the whole detection area. Schematic drawing of the scanning algorithm is depicted in Fig. 1.

In fact, with this approach the image pixel field is formed by temporal sequence of photodetector positions, and the pixel size is determined by the aperture of the photodetector sensor. This "temporal

*papoyan@ipr.sci.am 


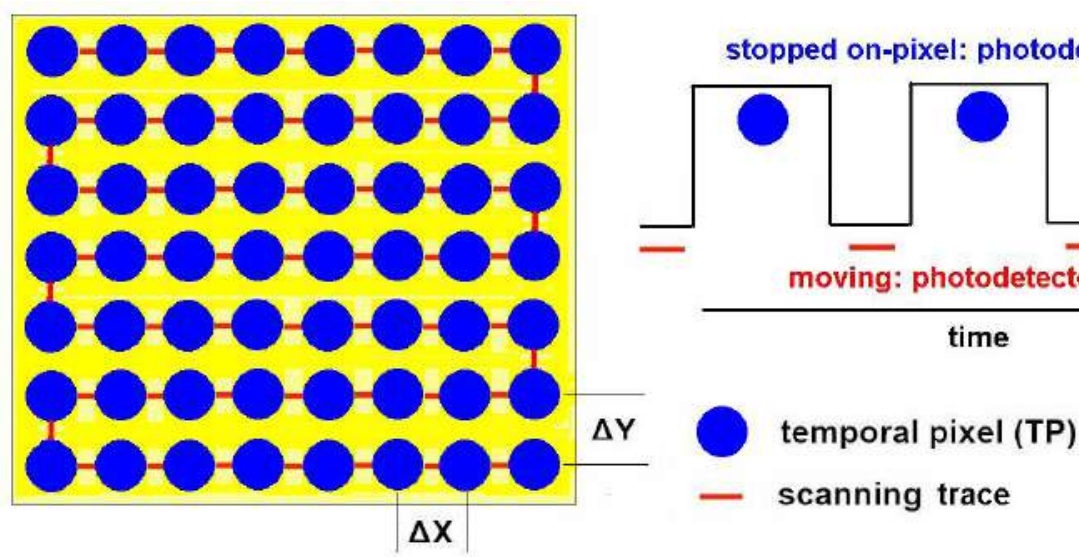

Figure 1: Schematic drawing of the scanning algorithm (see text).

pixels" (TP) touch each other, forming a filled view area. The most intuitive trace for scanning is lineby-line movement, with direction reversal on each subsequent line (see red lines in Fig. 1). Scanning is realized by "jump-and-stop" principle: on each pixel, photodetector stops for defined time allowing readout and integration of the signal, then it jumps to the neighboring pixel position, with readout off (integration reset), then the cycle repeats (see a right-side diagram in the figure).

The entire course of the scan can be easily controlled by a computer program: one can set the scan step (spatial resolution) along the $\mathrm{X}$ and $\mathrm{Y}$ axes, the jump time from pixel to pixel, the stop (readout) time on each pixel; it is also possible to change the scanning trace or select a separate area with the given position and sizes for detailed study.

Despite the apparent complexity, this approach to image construction has several advantages compared to the generally accepted method of recording images with CCD or CMOS cameras. Firstly, it allows to easily set the integration time of the signal from each pixel, and hence the sensitivity. This is especially important for astronomical applications, where the studied objects are relatively stationary. Secondly, since each pixel is formed by the same photodetector, the dispersion of parameters in pixels inherent in matrix detectors is outright eliminated. Thirdly, it gives flexibility to vary scanning parameters to user demand, which can be helpful for real observation (for example, fast preliminary search and identification of the proper view field with low resolution, followed by high-resolution recording). Finally, this method allows extension of the effective imaging sensor size at user demand (up to the size of astronomical photographic plates), preserving spatial resolution (pixel per mm) invariable.

Another advantage of the scanning technique is the data acquisition principle, which facilitates handling and processing of recorded information. As the measurement data is being recorded in series, as a temporal sequence synchronized with the scanning, it is straightforward to implement various data manipulation methods, such as elimination of background illumination, contrast enhancement, highlighting moving objects in an image (by removing still ones), etc. These enhancements can be realized, in particular, by subtraction of data of neighboring pixels in the same frame, and subtraction of data from the same pixels in consecutive frames. Such data processing techniques have been successfully implemented in transmission imaging for revealing objects embedded in highly-scattering and absorbing matrix environment (Vardanyan et al. (2010), Vardanyan et al. (2011)).

Besides the above mentioned advantages, the proposed scanning imaging approach has also some drawbacks, such as mechanical motion in the detection system and slowness of image recording. Nevertheless, the proposed technique can be considered as a real alternative to the existing approach to astronomical photography. In the next section, we will analyze in detail the key parameters and characteristics of the individual units of the proposed device to justify its applicability in astronomical observations. 


\section{Technical implementation and performance estimates}

In this section we will consider the general scheme of the device, the main requirements and operation parameters for its different components, and technical feasibility of the proposed imaging approach.

Direct placement (mounting) of photodetector on the scanning head is not preferable for several reasons. First, for photodetectors, the sensor aperture serving as effective detection pixel is unacceptably large (typically $>100 \mu \mathrm{m}$ ). Second, mounting a relatively heavy opto-electronic component with wiring on a movable scanner head may cause mechanical vibration and non-proper operation of the actuators. Third, it is difficult to provide proper thermal stabilization of the photodetector and its immunity against ambient electric noise when it is moving.

As an appropriate solution can be delivery of light to an unmovable photodetector using an optical fiber with a termination mounted on an actuator-driven 2D translation stage (see Fig. 2). The most appropriate for this is pigtailed photodiode with flat cleave termination. In this configuration, the diameter of optical fiber core rather than photodetector sensor size determines the size of effective pixel. We should note that single-mode fibers have much smaller core diameters than multi-mode fibers. Typical single-mode optical fibers (SMF) carrying only a single mode of light, the transverse mode, consist of a core $(\oslash 5 \mu \mathrm{m})$, a cladding $(\oslash 125 \mu \mathrm{m})$, a buffer $(\oslash 250 \mu \mathrm{m})$, and a jacket $(\oslash 900$ $\mu \mathrm{m})$. Therefore, the use of a SMF with flat cleave termination makes it possible to attain spatial physical resolution of 200 pixels/mm.

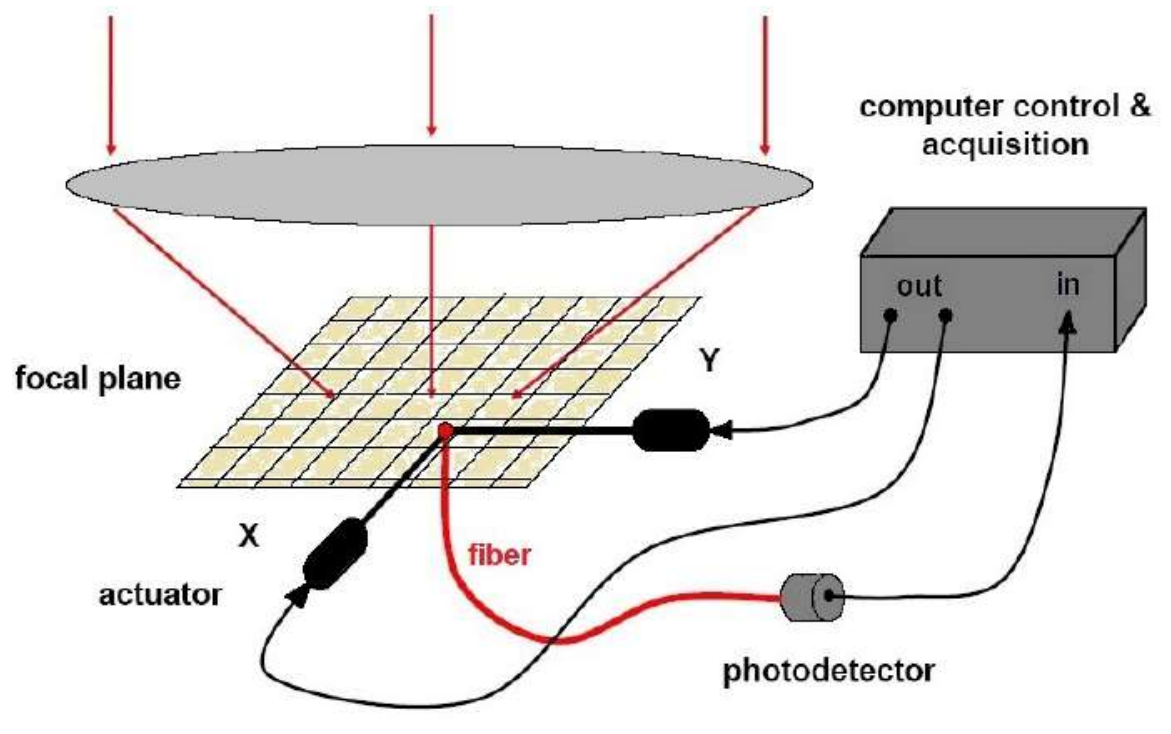

Figure 2: Schematic drawing of the detection system.

Let us now briefly discuss operation characteristics of the single-frequency fibers. Among the most important parameters is the Numerical Aperture $(N A)$ of a fiber, which is defined as the sine of the largest angle the incident ray can have for total internal reflectance in the core. A higher core index, with respect to the cladding, means larger $N A$. Rays launched outside the angle specified by a fiber's $N A$ will excite radiation modes of the fiber, which will impose losses. $N A$ also indicates how easy it is to couple light into a fiber. Another important parameter is the "normalized frequency parameter of a fiber", also called the $V$ number, mathematically expressed as

$$
V=\frac{2 \pi a}{\lambda} N A=\frac{2 \pi a}{\lambda} \sqrt{n_{\text {core }}^{2}-n_{\text {clad }}^{2}},
$$

where $a$ is the fiber core radius, $\lambda$ is light wavelength, $n_{\text {core }}$ is the index of refraction of the core, $n_{\text {clad }}$ is the index of refraction of the cladding $\left(n_{\text {core }}>n_{\text {clad }}\right)$ Hidehiko et al. (2006). Many fiber parameters can be expressed in terms of $V$, such as: the number of modes at a given wavelength, mode cut off conditions, and propagation constants. For example, the number of guided modes in a step index multimode fiber is given by: $M \approx V^{2} / 2$, and a step index fiber becomes single-mode for a given 
wavelength when $V<V_{c}=2.405$. The value of $V_{c}$ determines also the cutoff wavelength of the fiber $\lambda_{c}$, the minimum wavelength for which a particular fiber still acts as a single-mode fiber. Above the cutoff wavelength, the fiber supports a single mode, while below it becomes multimode. Knowing $V_{c}$, the value of $\lambda_{c}$ can be determined using Eq. 1.

Pigtailed photodiodes couped to patch fiber cables with flat cleave termination are commercially available for different wavelengths ranged from visible to near-infared. The single-mode wavelength bandwidth of these fibers in visible reaches up to $150 \mathrm{~nm}$. In order to avoid light input losses, the fiber tip should be antireflection coated in operation wavelength range. In any case, we should note that the proposed approach intrinsically implies monochrome imaging. However, spectral decomposition of the recorded light from each "time pixel" is also possible. When using a single-mode fiber, spectral analysis can be carried out in a relatively narrow range of supported wavelengths (see above). For spectral studies, dispersing elements as well as narrow-band filters can be used.

Two-dimensional spatial scanning of the fiber tip in the focal plane can be performed by backlashfree actuators based on stepper motor-controlled linear translation stages, which allow computer control of the scanning trace and speed. Such devices meeting the set requirements are commercially available. For example, FCL50 from Newport provides $50 \mathrm{~mm}$ travel range with $20 \mathrm{~mm} / \mathrm{s}$ speed, $150 \mathrm{~nm}$ incremental motion, $\pm 1 \mu \mathrm{m}$ accuracy and bi-directional repeatability, and axial (lateral) load capacity of $40 \mathrm{~N}$ sufficient to carry the optical fiber.

Now let us consider the next key element of the scheme, the photodetector. Independently of the type of light sensors (matrix, or array, detectors such as CCD or SMOS, opposed to solitary, or point detectors such as photodiodes), detection of light can be decomposed in three simplified steps: i) conversion of light to free electrical charge-carriers; ii) conversion of current to voltage; and iii) measurement of voltage. Both types of photodetectors are characterized by the following figures of merit - common parameters to quantify their operation: responsivity, quantum efficiency, noise-equivalent power, specific detectivity, and response time (see e.g. Posch (2010)).

1) Responsivity $R_{\lambda}$ measured in $\mathrm{A} / \mathrm{W}$ denotes for given wavelength the input-output relationship, or gain of a detector, and is defined as the ratio of light-induced photocurrent density $J_{p h}$ and the incident optical power per unit area $P_{0}$ :

$$
R_{\lambda}=\frac{J_{p h}}{P_{0}}
$$

Responsivity of Si-based detectors can reach maximum value of $\sim 0.8 \mathrm{~A} / \mathrm{W}$ at $\lambda \sim 1000 \mathrm{~nm}$.

2) Quantum efficiency $\eta$, usually given in percent, is defined as the number of photon-generated free charge carriers $\left(J_{p h} / q\right.$, where $q$ is the electron charge) per impinging photon (the number of photons is $P_{0}$ divided by the energy of one photon $h \nu$ ):

$$
\eta=\frac{J_{p h} / q}{P_{0} / h \nu}=R_{\lambda} \frac{h \nu}{q} .
$$

Although quantum efficiency of some state-of-the-art detectors may exceed $90 \%$, the real values are usually smaller. Thus, for Hubble Space Telescope's Wide Field and Planetary Camera $2, \eta \approx 35-$ $40 \%$ in the spectral range of $600-800 \mathrm{~nm}$.

3) The noise-equivalent power $(N E P)$ measured in $\mathrm{W} / \sqrt{\mathrm{Hz}}$ is defined as the intensity of incident light required to generate a current equal to the noise current $j_{n}$, or yielding a signal-to-noise ratio (SNR) of unity in a $1 \mathrm{~Hz}$ frequency bandwidth:

$$
N E P=j_{n} / R_{\lambda}=\frac{\sqrt{\sum j_{n i}^{2}}}{R_{\lambda}} .
$$

The lower limit of light detection is determined by the noise characteristics of the device of different origin $\left(j_{n i}\right)$. The typical photodetector noise is the sum of thermal noise and shot noise from dark current and photocurrent. For commercial photodiodes, the value of $N E P$ can be as low as $5 \times 10^{-16}$ $\mathrm{W} / \sqrt{\mathrm{Hz}}$. It is important to note that for matrix detectors such as CCD the value of $N E P$ is always higher because of additional noise contribution from the "read noise". 
4) Specific detectivity $D^{*}$ measured in Jones units $(\mathrm{cm} \cdot \sqrt{\mathrm{Hz}} / \mathrm{W})$ is defined as the ratio of detector area $S_{d e t}$ and noise frequency bandwidth $\Delta f_{n}$ to the $N E P$ :

$$
D^{*}=\frac{\sqrt{S_{d e t} \Delta f_{n}}}{N E P} .
$$

Specific detectivity of modern silicon PIN photodiodes can be as high as $10^{13}$ Jones.

5) The response time (or rise/fall time) quantifies the response of photodetector to abrupt change of photon flux. Silicon PIN photodiodes allow fast detection with rise/fall time below 0.1 ns. For CCD cameras, the readout is the slowest step in the image acquisition. A ms-range integration time is typical for most of matrix (array) detectors.

Summarizing, one can see that despite the obvious convenience of using CCD cameras, which allow to directly obtain an image of the object under study, their performance characteristics are generally inferior to solitary (point) photodetectors, in particular silicon PIN photodiodes. The drawbacks of matrix detectors mainly originate from the readout (additional noise contribution and slower operation). Moreover, for real matrix imaging detectors, the performance characteristics somewhat vary from pixel to pixel, which may cause overall image distortion.

Commercially available silicon pigtailed PIN photodiodes coupled with 4-5 $\mu$ m-diameter and $N A$ $=0.13$ single-mode fibers (e.g. FDSP series from Thorlabs) provide noise equivalent power of $3 \times 10^{-15}$ $\mathrm{W} \cdot \mathrm{Hz}^{-1 / 2}$ with $700 \mathrm{ps}$ rise/fall time and $0.01 \mathrm{nA}$ dark current, available for $610-770$ and $780-970$ nm wavelength ranges.

Finally, let us consider the functionality of the computer control \& acquisition device. It comprises three main structural units: i) a computer with installed control/acquisition software and user desktop interface; ii) a multifunctional analog/digital input/output (DAQ) device serving as an interface between the computer on one side, and photodetector and actuator stepper motors on the other side; iii) signal conditioning circuits, which allow to adopt the operation voltages and currents of photodetector and stepper motors to the input/output signal levels of DAQ device.

Control software installed in the computer designs and sends to DAQ device a multichannel sequence of phase-shifted pulses needed to control the operation of actuator stepper motors. Control pulses formed at the 8 output channels of DAQ device (by 4 for each motor) undergo power amplification at the output signal conditioning unit, which feeds $\mathrm{X}$ and $\mathrm{Y}$ actuator stepper motors. Translation trace, step, speed, and stopping (data acquisition) time at each temporal pixel are software-controlled with user desktop interface.

In turn, the photodiode signal generated during the stopping (on-pixel) periods enters the operation amplifier serving as input signal conditioning circuit to match the DAQ analog input voltage. After analog-to-digital conversion, the digitized signal enters the computer. As scanning control and data acquisition channels are mutually synchronized via a unique system clock, building of image frame after completion of scanning cycle (or even in the course), is rather straightforward.

Hardware solutions for structural elements of the computer control \& acquisition device are well elaborated and widely available. There are no special requirements for a computer. It can be a desktop PC, a notebook, or even a low-cost Raspberry Pi single-board computer. It just has to support the control software of a multifunctional analog/digital input/output (DAQ) device (e.g. LabView if National Instruments DAQ device is chosen).

A DAQ device should support at least 8-channel digital outputs (DO) for the control of stepper motors and at least one analog input (AI) channel for recording a photodetector signal (preferably with at least 24 bit resolution and $100 \mathrm{kS} / \mathrm{s}$ sample rate). Many companies produce such devices. We should note that if commercial translation stages are used as actuators, such as FCL Series from Newport, they can be connected to computer directly via USB port, without a need of a 8-channel digital outputs, and DAQ board with a single AI channel will be sufficient.

Signal conditioning circuit to drive stepper motors is a simple power amplifier scheme converting low-current logic high/low voltage signal to control pulses needed to operate the chosen motors. As for conditioning the input analog signal from the photodetector, a low-noise linear voltage amplifier should be used, which will allow you to match the voltage range from the photodiode with the working range of the input channel of the DAQ device. Both the output and input signal conditioning circuits are commercially available, but they can be also easily home made. 


\section{Conclusions}

To summarize, we have considered applicability of the method of pointwise image formation for astronomical focal plane photography. A practical scheme of the imaging device is proposed, its achievable characteristics and parameters are estimated based on realistic technical solutions and available structural units.

The use of a flat cleave core termination of the single-mode optical fiber as a "temporal pixel" allows to attain pixel size of $\oslash 5 \mu \mathrm{m}$. Such fibers coupled with silicon pigtailed fast PIN photodiodes operating in visible and near infrared and having noise equivalent power of $3 \times 10^{-15} \mathrm{~W} \cdot \mathrm{Hz}^{-1 / 2}$ are commercially available. Also stepper motor driven translation stages supporting $5 \mu \mathrm{m}$ scanning step with $\pm 1 \mu \mathrm{m}$ precision are available. Supposing $50 \mu$ s integration time per pixel, which is sufficient for sensitive detection of astronomical objects, recording of image from 1 megapixel area $(5 \times 5 \mathrm{~mm})$ will last $\approx 1$ minute. Important to note that effective sensor size for this approach is limited by the travel range of actuators (translation stages), and in principle can match the size of standard astronomical photographic plates.

Recording of images is fully computer-controlled and customizable on user remand, realized with the use of available DAQ devices and elaborated software.

\section{References}

Hidehiko Y., Polynkin P., Mansuripur M., 2006, Journal of Lightwave Technology, 24, 1350

Posch C., 2010, "Detectors, Pixels, and Signal Processing", Chapter 4 in: Smart Cameras (Ed. A.N. Belbachir). Springer, ISBN: 978-1-4419-0952-7

Vardanyan K., Khachaturova A., Varzhapetyan S., Badalyan A., Shmavonyan S., Papoyan A., 2010, Optoelectronics and Advanced Materials- Rapid Communications, 4, 1163

Vardanyan K., Khachaturova A., Varzhapetyan S., Badalyan A., Shmavonyan S., Papoyan A., 2011, Proceedings of SPIE, 7998, 799814 